\title{
Feature Selective Validation of Automotive EMC Pre-compliance Tests
}

\author{
Vaclav RUZEK ${ }^{1,2}$, Jiri DRINOVSKY ${ }^{2}$, Jan CUPAK ${ }^{2}$ \\ ${ }^{1}$ EMC Dept., SKODA AUTO, a.s., Vaclava Klementa 869, 29301 Mlada Boleslav, Czech Republic \\ ${ }^{2}$ Dept. of Radio Electronics, Brno University of Technology, Technicka 12, 61200 Brno, Czech Republic \\ vaclav.ruzek@skoda-auto.cz,drino@feec.vutbr.cz,xcupak02@stud.feec.vutbr.cz
}

Submitted November 6, 2017 / Accepted December 5, 2017

\begin{abstract}
The pre-compliance tests of electromagnetic immunity are at present a crucial issue for all major automotive manufacturers. In current practice, there are two basic ways to implement these tests. The first one is based on significant simplification of measurement methods and the subsequent transformation of results for relevant estimation of certification measurement results. The second is based on the application of numerical methods and the calculation of the electric field intensity distribution in the car body. The question, however, remains the degree of correlation between these methods, especially when confronting the results of certification measurement. This article provides an overview of the results obtained using both methods and offers an innovative view of the method of comparison using the FSV.
\end{abstract}

\section{Keywords}

Feature Selective Validation, pre-compliance testing, automotive EMC, results processing

\section{Introduction}

Numerical simulations have become an integral part of serial production preparation in all sectors of the automotive industry, electromagnetic immunity including. Numerical simulation allows to significantly shorten product development time and reduce the costs of performed physical tests.

The open question is the degree of numerical methods validity with respect to real test results. This leads to the decision whether it is possible to completely replace real measurements by numerical simulations, or use them as a way of certification [1]. The vast majority of numerical methods currently used for numerical simulation of electromagnetic problems are based on Maxwell's equations. Their overview can be found in [2].

In the same way, high hopes are introduced [3] into the modification of the established measurement procedures in order to simplify and implement them with low- cost testing equipment. Such an approach requires a thorough knowledge of the method, as well as a method for matching the results obtained by means of pre-certification measurements to the certification results.

An analysis of available measurement methods [4], [5] shows that we have the following primary test procedures:

- Tests based on the antenna method (ALSE) [6],

- Current pulse injection (BCI) tests [7], [8], [9].

In both cases, we must consider ways to modify the method to simplify, reduce and accelerate tests.

\subsection{Feature Selective Validation Method}

Feature Selective Validation Method (FSV) is a modern, very effective and objective method, particularly suitable for verifying the correlation of data obtained using CEM (Computational Electro Magnetism) methods and validation measurements [10], [11]. The basic motivation for the application of this method to technical practice is an effort to quantify:

- Repeatability of measurement,

- Measurement portability,

- Effect of the operator,

- Method variation.

The basis of the FSV technique is to decompose the results into two components and then recombine the results to obtain an overview of the overall consistency. The components used are the Amplitude Difference Measure (ADM), which compares the amplitudes and trends of individual datasets (1), and the Feature Difference Measure (FDM) that compares rapidly variable characters (2) (as a function of an independent variable) of these sets. AMD and FDM are then combined into the GDM (3) form. All ADM, FDM, and GDM components are usable as a pointby-point analysis or as a single global measurement [12]. For the ADM, FDM, and GDM values, we can use the following equations (1), (2), (3). 


$$
\begin{gathered}
A D M(f)=\left|\frac{\alpha}{\beta}\right|+\left|\frac{\chi}{\delta}\right| \exp \left|\frac{\chi}{\delta}\right|, \\
F D M(f)=2\left(\left|F D M_{1}(f)+F D M_{2}(f)\right|\right), \\
G D M=\sqrt{A D M(f)^{2}+F D M(f)^{2}}
\end{gathered}
$$

where $\alpha, \beta, \chi, \delta$ are calculation elements of the individual difference functions (DC and Lo) and $\mathrm{FDM}_{1}, \mathrm{FDM}_{2}$, are elements of the inverse transformation of Hi and Lo passages. GDM is an overall scale compliance of ADM and FDM. A detailed derivation of equations can be found in [11].

By computing the FSV, we obtain the relationship between the numerical output and its distribution in individual categories, with the number of validated data points placed in the individual categories being preferably expressed using the histogram [13], [14]. In Tab. 1 we can see an interpretive scale capturing the quantitative and qualitative aspects of FSV values that this method can acquire.

In this context, the goal of this paper is to present a methodology for the validation of numerical results and experimental measurement that includes the following.

1) Provide basic rules for numerical modeling in the automotive EMC area (model simplification and division into functional parts).

2) Describe an evaluation methodology (E field intensity measurements, choice of proper measurement points).

3) Provide a novel test setup for experimental measurement and set rules for vehicle body dismantling.

4) Discuss the Feature Selective Validation method application in the case of automotive industry.

\section{Pre-compliance Immunity Test Based on Numerical Simulation}

As mentioned above, there are two basic ways of obtaining pre-certification data describing the electric field intensity distribution. The most promising is the application of numerical simulations. In order to assess their results, we firstly perform a theoretical analysis of the resonant behavior of the car body, followed by practical calculations.

\begin{tabular}{|c|c|}
\hline $\begin{array}{c}\text { FSV value } \\
\text { (quantitative) }\end{array}$ & $\begin{array}{c}\text { FSV interpretation } \\
\text { (qualitative) }\end{array}$ \\
\hline Less than 0.1 & „Excellent“ \\
\hline Between 0.1 and 0.2 & „Very good“ \\
\hline Between 0.2 and 0.4 & „Good“ \\
\hline Between 0.4 and 0.8 & „Fair“ \\
\hline Between 0.8 and 1.6 & „Poor“ \\
\hline Larger than 1.6 & „Very poor“ \\
\hline
\end{tabular}

Tab. 1. The FSV interpretation measure.

\subsection{Estimated Field Distribution in Car Chassis}

The own resonant frequency, the resonator quality, and especially the resonant frequency change, can be seen as a result of several failure types. From the point of view of this article, we will describe the change of resonant frequency and resonator quality caused by the dielectric material placed inside the resonator. For the description of this situation, we will advantageously use the Slater failure method [15].

The description is based on the ideal situation when the resonator is perfectly closed, its walls have an ideal (infinite) conductivity and there is a perfectly homogeneous environment in the volume of the resonator. In such an environment, the distribution of the electromagnetic field can be found by the analytical method. Assuming that, we start from homogeneous Maxwell equations, we obtain wave equations for the partial components of the electromagnetic field

$$
\begin{aligned}
\nabla^{2} \boldsymbol{E}_{a}+k_{a}^{2} \boldsymbol{E}_{a} & =0, \\
\nabla^{2} \boldsymbol{H}_{a}+k_{a}^{2} \boldsymbol{H}_{a} & =0
\end{aligned}
$$

where $k_{a}$ is the wave number, $\boldsymbol{E}_{a}$ and $\boldsymbol{H}_{a}$ the function denoting the distribution of the intact field for individual modes. These equations can have an infinite number of solutions based on an infinite number of $k_{a}$ values and corresponding boundary conditions.

For $\int \boldsymbol{E} \cdot \boldsymbol{E}_{a} \mathrm{~d} V$ and $\int \boldsymbol{H} \cdot \boldsymbol{H}_{a} \mathrm{~d} V$ we get a waveform, in this case a non-zero right side. The meaning of the individual members on the right side of the equation is the following: In analogy with simple harmonic movement, the external forces represent an element that changes the resonant frequency of the oscillator. Thus we obtain a modified electromagnetic field and thus the resonance frequency to the intact state [15]. This is affected by:

a) Currents flowing through the resonator,

b) Holes in the walls of the resonator (imperfect resonant cavity);

c) The final conductivity of the resonator walls (caused by a non-zero tangential component).

According to Slater's method we use the analogy with resonant circuit defined by RLC parameters. For the resonant frequency the following equation can be found

$$
\mathrm{j}\left(\frac{\omega}{\omega_{0}}-\frac{\omega_{0}}{\omega}\right)+\frac{1}{Q}=0
$$

where $\omega_{0}$ is the resonant frequency of the LC circuit and $Q$ is the quality factor. For further consideration, we will consider that the angular frequency $\omega_{0}$ does not differ much from one of the $\omega_{a}$ resonance frequencies. Another derivation [15] gives us a relation (7) by which we can easily express changes in the resonance frequency given by the above effects: the currents flowing through the resona- 
tor, the holes in the resonator walls and the final conductivity of the resonator.

$$
\begin{aligned}
\frac{1}{Q}-2 \mathrm{j} \frac{\Delta \omega_{a}}{\omega_{a}}= & -\frac{\mathrm{j}}{\omega_{a} \sqrt{\varepsilon_{0} \mu_{0}}} \frac{\int_{S}(\boldsymbol{n} \times \boldsymbol{E}) \cdot \boldsymbol{H}_{a} \mathrm{~d} A}{\int \boldsymbol{E} \cdot \boldsymbol{E}_{a} \mathrm{~d} V}- \\
& \frac{1}{\varepsilon_{0} \omega_{0}} \frac{\int_{S^{\prime}}(\boldsymbol{n} \times \boldsymbol{H}) \cdot \boldsymbol{E}_{a} \mathrm{~d} A}{\int \boldsymbol{E} \cdot \boldsymbol{E}_{a} \mathrm{~d} V}+\frac{1}{\varepsilon_{0} \omega_{a}} \frac{\int \boldsymbol{J} \cdot \boldsymbol{E}_{a} \mathrm{~d} V}{\int \boldsymbol{E} \cdot \boldsymbol{E}_{a} \mathrm{~d} V} .
\end{aligned}
$$

Equation (7) can be considered as a fundamental expression of changes in the properties of the resonator. The equation can be applied now to the cavity resonator environment of the vehicle body. In this setup, we have to take into consideration the following conditions, which distinguish the ideal resonator from the described real one:

a) The resonator is made up of a material with a finite conductivity.

b) In real conditions, the walls of the resonator are covered by a layer of dielectric materials (coating materials, foam-absorbing material).

c) The resonant cavity, due to its irregularity, allows the emergence of certain resonant modes only in certain parts of it.

d) The resonator has a large number of holes, significantly reducing its quality.

\subsection{Field Distribution Obtained Using Nu- merical Simulations}

Based on the performed experiments, the MoM analysis method was chosen as the most appropriate [16]. The ReMesh tool [17] was used for the model preparation. For the solution of the equation system, the 3D TriD field solver, which forms the core part of the EMC Studio program package [18], was used.

The most important aspect of the chosen approach is the thorough preparation of the numerical model, based on well-available CRASH models. Modification of such a model consists of the following steps:

- reducing the extreme redundancy of the original model data,

- removing excess surfaces of the reduced model,

- appropriate division of the model into functional units,

- definition of model network size.

Based on the above described strategy, the model is divided into two basic parts:

- Welded and mounted car body model - Fig. 1,

- Other inserted parts (aggregate, front axle, dashboard reinforcement and seat structure) - Fig. 2.

The experiment is performed by the exposure of the prepared model using a vertically polarized plane wave at the most critical 30 to $220 \mathrm{MHz}$ band. From practical experience we know that exceeding the electric field strength

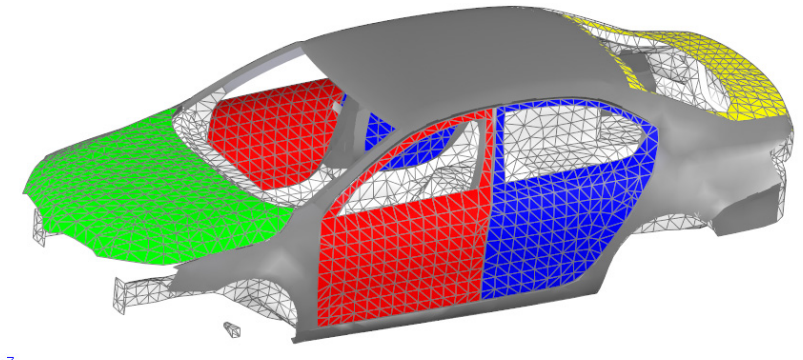

$\stackrel{P^{2}}{\longrightarrow}$

Fig. 1. The basic vehicle body model. Color-coded body elements.

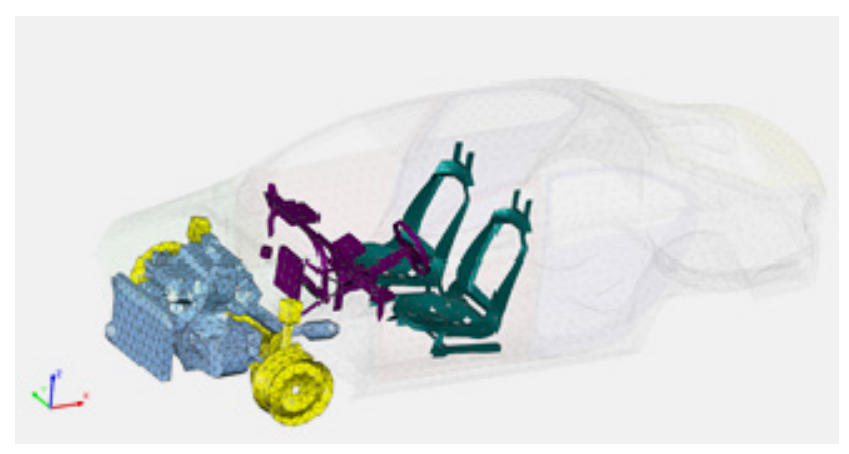

Fig. 2. Supplementary parts of the numerical model geometry.

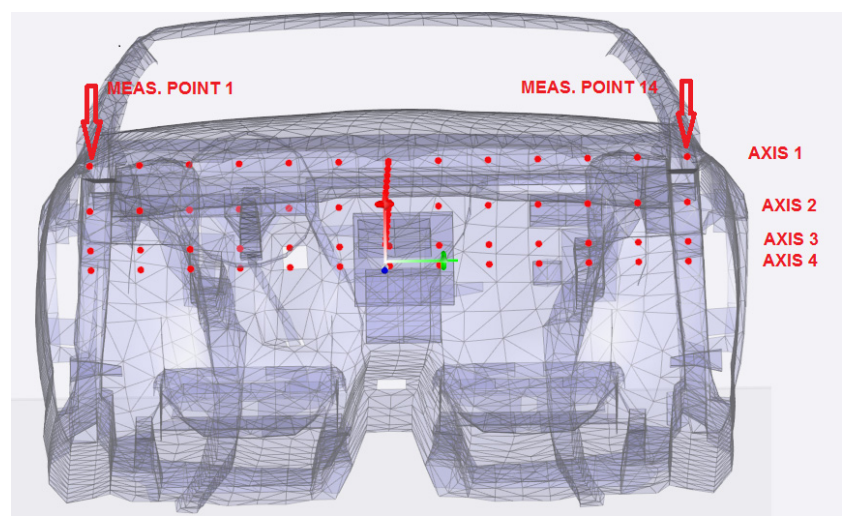

Fig. 3. Location of the electric field probe array in the car body.

inside the body over a $100 \mathrm{~V} / \mathrm{m}$ limit can cause problems in the proper operation of CAN bus communication, sensors and controls unit. For this reason, we will consider $100 \mathrm{~V} / \mathrm{m}$ as the critical limit.

In order to measure the electric field strength, an array of virtual electric field probes was placed in the body space according to Fig. 3 .

The probe matrix was placed on the dashboard level based on theoretical analysis. Individual points were placed in the axes and, according to their vertical position, were numbered 1 to 14 from the left side. The E1-E5 point groups then represent the steering wheel area, the E6-E9 area of the center console and the E10-E14 passenger airbag area.

If the model is exposed by vertically polarized plane wave (Fig. 4), we can clearly identify the dominant reso- 
nant frequency of $71 \mathrm{MHz}$ in the group of measuring points E1-E5, which manifests at all points in this group. It is the lowest self-contained resonant mode of the interior of the bodywork with a projection for all measuring axes. Intensity of the electric field detected especially at points E2-E4 exceeds $500 \mathrm{~V} / \mathrm{m}$. Such high intensity is mainly caused by the contributions of surface currents flowing over the ideally conductive (PEC) elements of the net forming the steering wheel assembly. However, given the enormous intensity of the electric field, we do not consider this result to be completely accurate - we expect the real intensity around $250 \mathrm{~V} / \mathrm{m}$. There is also a shift away from the trend in E1. This point is significantly influenced by its proximity to the body edge and thus the expected increase in the calculated field strength given by the computational method.

The area of points E6 to E9 (Fig. 5) shows, unlike the previous group, more expected results. Again, the dominant resonance frequency of $71 \mathrm{MHz}$ is shown here, supplemented additionally by the frequency of $120 \mathrm{MHz}$, which in the case of the previous group of points as resonant was reflected only marginally. The distribution of electric field strength in the area of points E10 to E14 (Fig. 6) is significantly influenced by two basic phenomena: the presence of controls on the dashboard reinforcement and the distribution of surface currents on the passenger door including the proximity of the door to the rest of the bodywork (high current density per segment).

The E12 and E13 points from the half of the monitored band clearly record the decrease in intensity caused by the weakening of the external bond. Point E14 is then affected by currents flowing along the body of the vehicle as well as point E1 and exhibits completely different behavior than the points located more inside the body. Due to the similarity of the results in individual groups, for future work we can consider only representative points usage in the given group (steering wheel, vehicle center, passenger airbag).

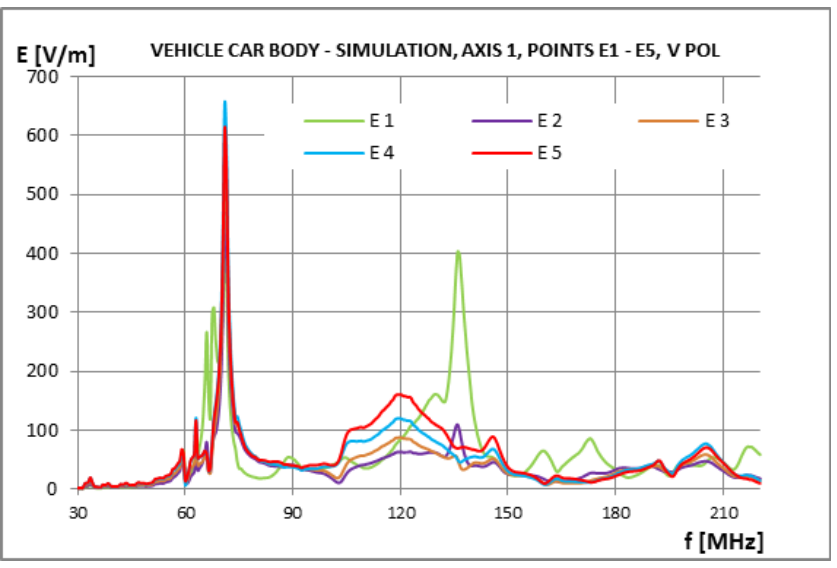

Fig. 4. The field strength obtained with the TriD at points E1 to E5.

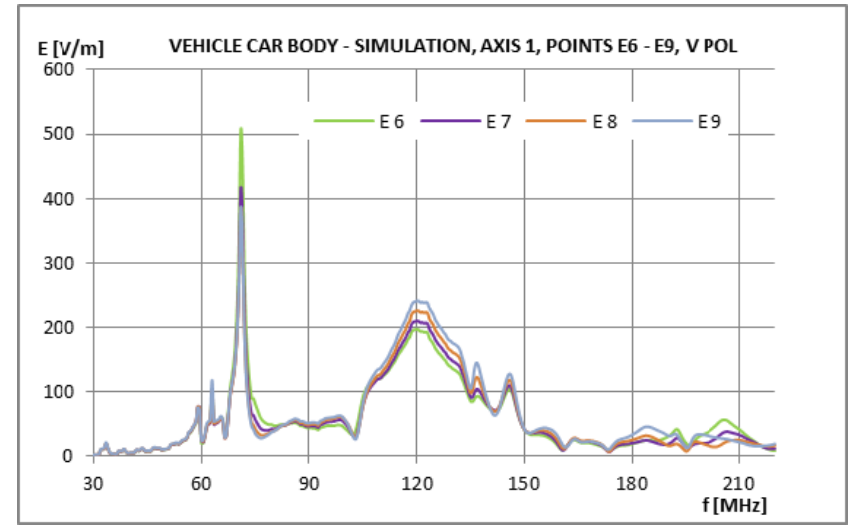

Fig. 5. The field strength obtained with the TriD at points E6 to E9.

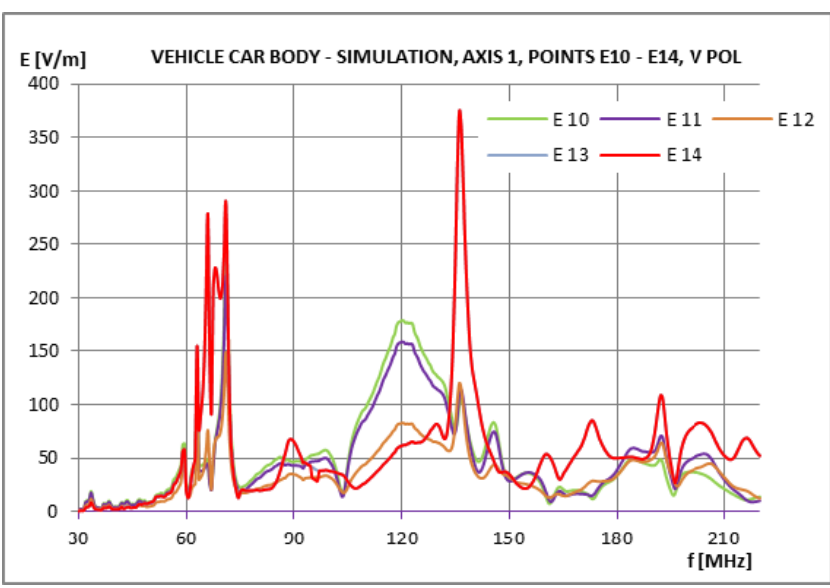

Fig. 6. The field strength obtained with the TriD at points E10 to E14.

\section{Experimental Measurement}

To verify the accuracy of numerical simulations, experimental measurements of the electric field strength were performed under the same conditions as for numerical simulation.

\subsection{Measurement Configuration}

For purposes of measurement, the body of the Škoda Octavia was dismantled according to the same rules as for the construction of the simulation model. The test car thus consists of the same parts that have been numerically modeled for simulation purposes.

The test was carried out by exposing the body of the vehicle to the field in the $30 \mathrm{MHz}$ to $220 \mathrm{MHz}$ frequency range with vertical and horizontal polarization. The intensity of the test field was chosen according to the same calibration curve as for numerical simulation with nominal intensity $\mathrm{E}=30 \mathrm{~V} / \mathrm{m}$. For the measurement, a semi-anechoic chamber was used. 


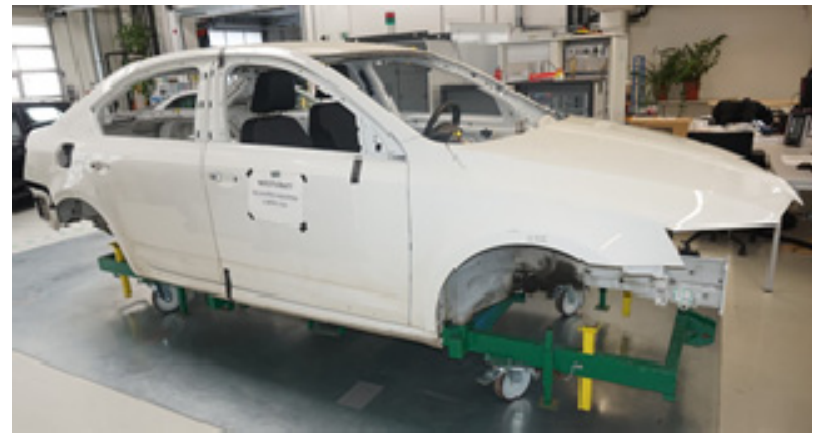

Fig. 7. Skoda Octavia car body after dismantle process.

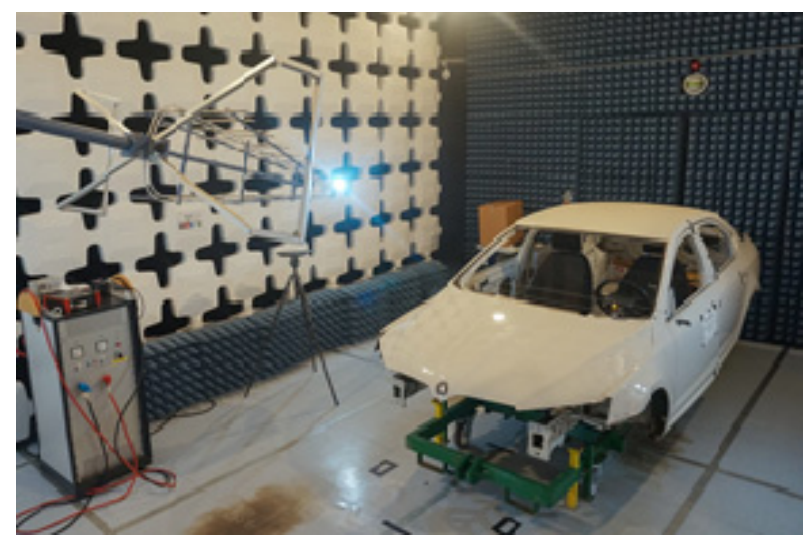

Fig. 8. Illustrative figure of the measurement.

\subsection{Obtained Results}

The methodology of measuring the intensity of the electric field was again based on the location of the individual measuring points in the matrix with the designation E1-E14, grouped again into 3 areas.

The measurement results obtained in axis 1 can be seen in Fig. 9 to Fig. 11. An overall view of the frequency response of the electric field indicates that for most of the tested frequencies, the intensity measured inside the vehicle is lower than the intensity of the calibration field. This means that the vehicle body forms an obstacle to the penetration of the wave into its interior space. Only localized resonant frequencies are the exception for this condition.

In all groups of measurement points, a dominant resonance at $78 \mathrm{MHz}$ is evident, especially when the body is exposed by a vertically polarized wave. The highest electric field strength $(310 \mathrm{~V} / \mathrm{m}$ measured at point E2) was achieved at this frequency in the steering wheel area, where individual measuring points are located in the direct vicinity of the conductive parts of the steering column reinforcement and the intensity of the electric field was greatly affected by currents flowing along their surface.

\section{Results Comparison Using FSV}

In order to express the degree of reliability and suitability of the proposed pre-certification simulation, a con-

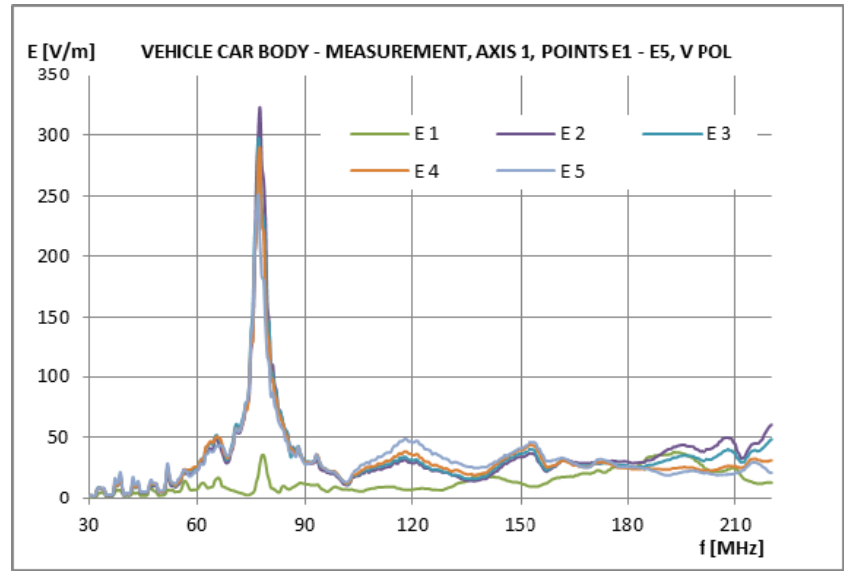

Fig. 9. The electric field strength measured in the bodywork at points E1-E5.

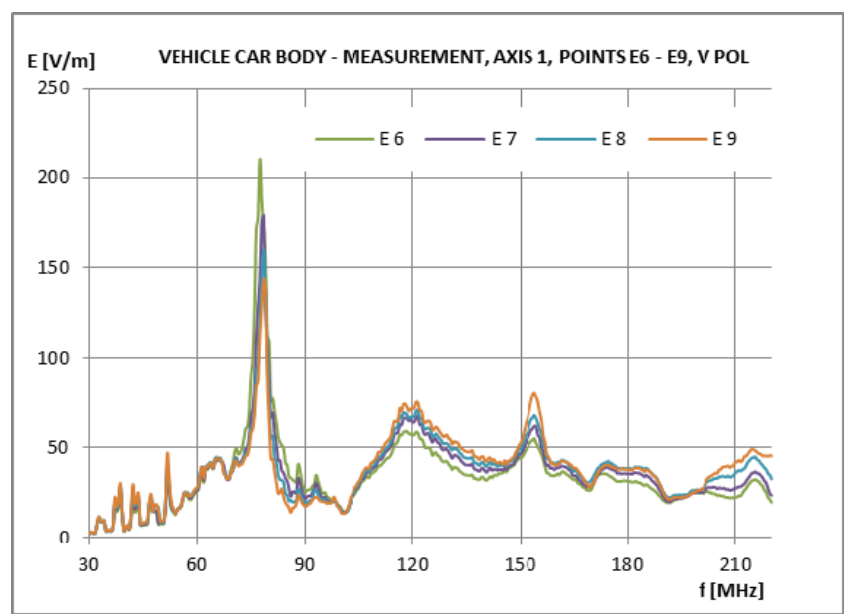

Fig. 10. The electric field strength measured in the bodywork at points E6-E9.

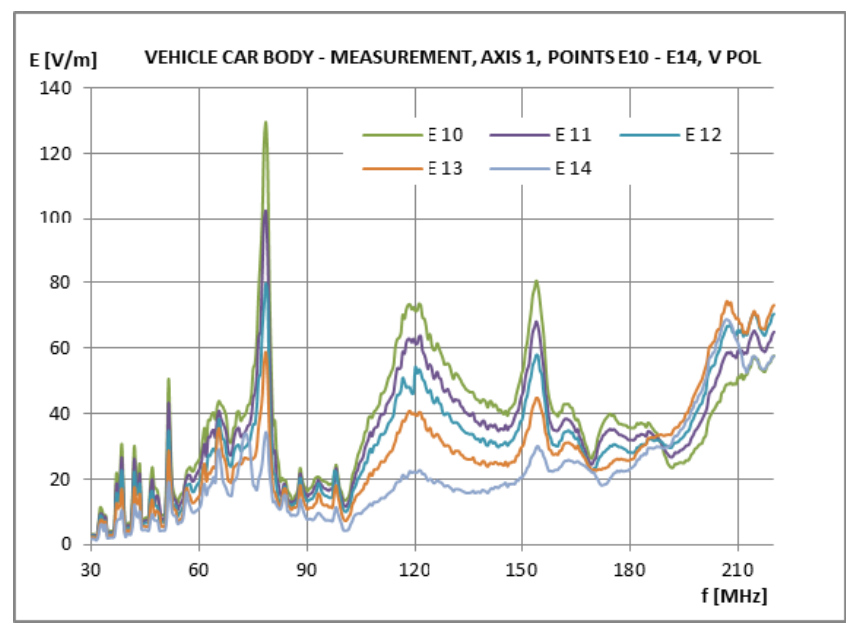

Fig. 11. The electric field strength measured in the bodywork at points E10-E14.

sistent comparison of the results obtained through individual approaches is necessary. For a better understanding of the difference interpretation before using the FVS method, the direct comparison of the numerical simulations results and the measured data is shown in Fig. 12 to 14 . The full 
line shows the results of the pre-certification measurements, the dashed line then shows the data obtained using numerical simulations.

In all sets of data we consider the values obtained in axis 1 and points E4, E7, E12, exposed by a vertically and horizontally polarized wave. The points above were chosen as a reference in the given area. Point E4 represents the electric field intensity distribution in the vicinity of the steering column and the steering wheel, the main resonant element of the vehicle interior in the frequency range being investigated. Point E7 allows the description of the intensity of the electric field in the area of the infotainment system display and represents essentially the center of the vehicle in its longitudinal plane. The last point E12 repre-

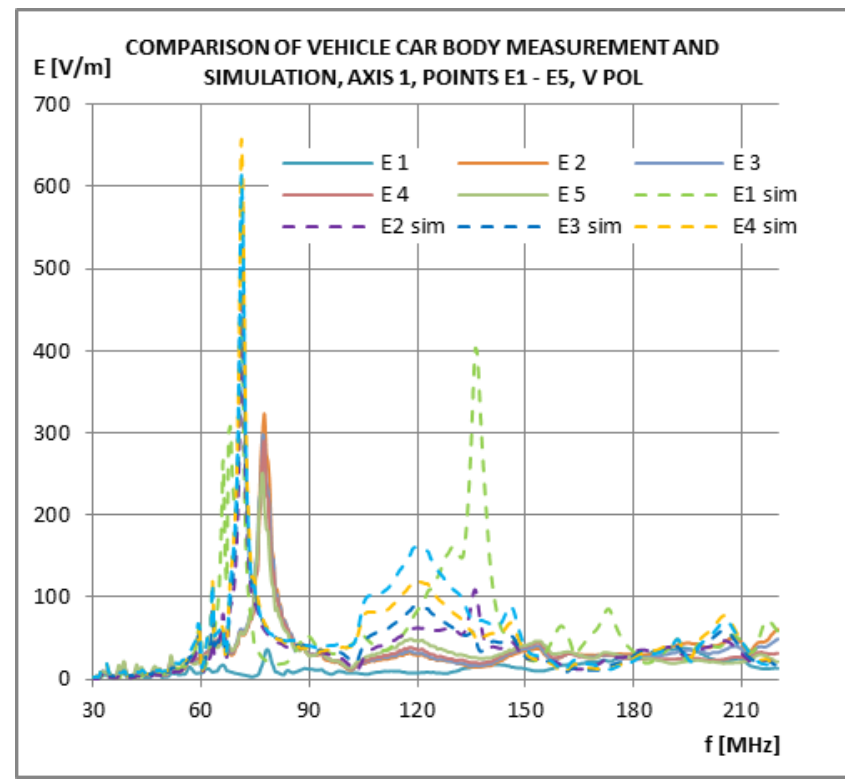

Fig. 12. Comparison of vehicle car body measurement and simulation for points E1-E5.

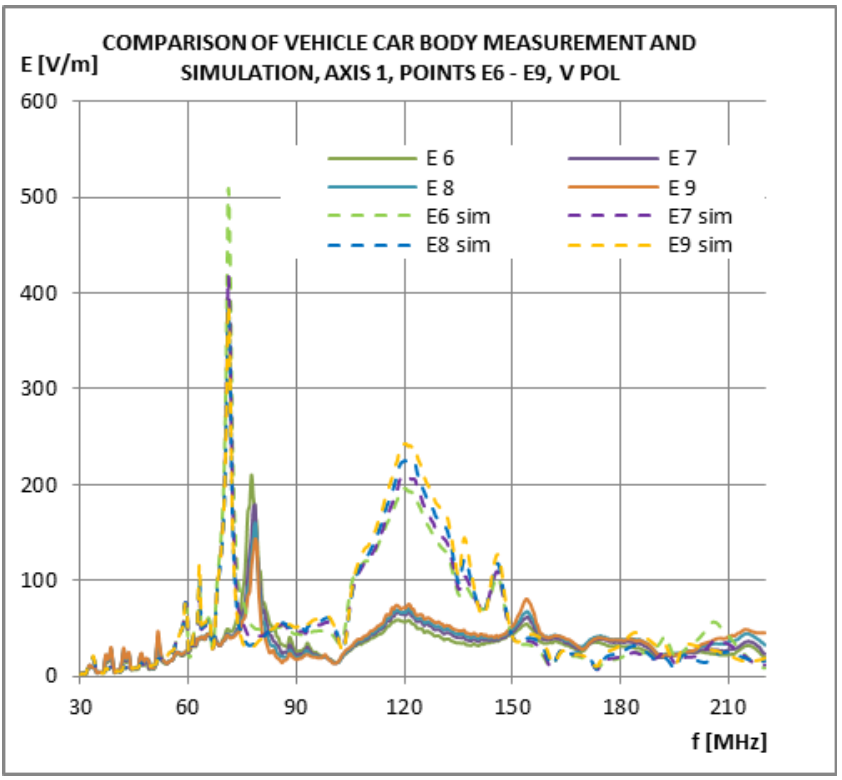

Fig. 13. Comparison of vehicle car body measurement and simulation for points E6-E9.

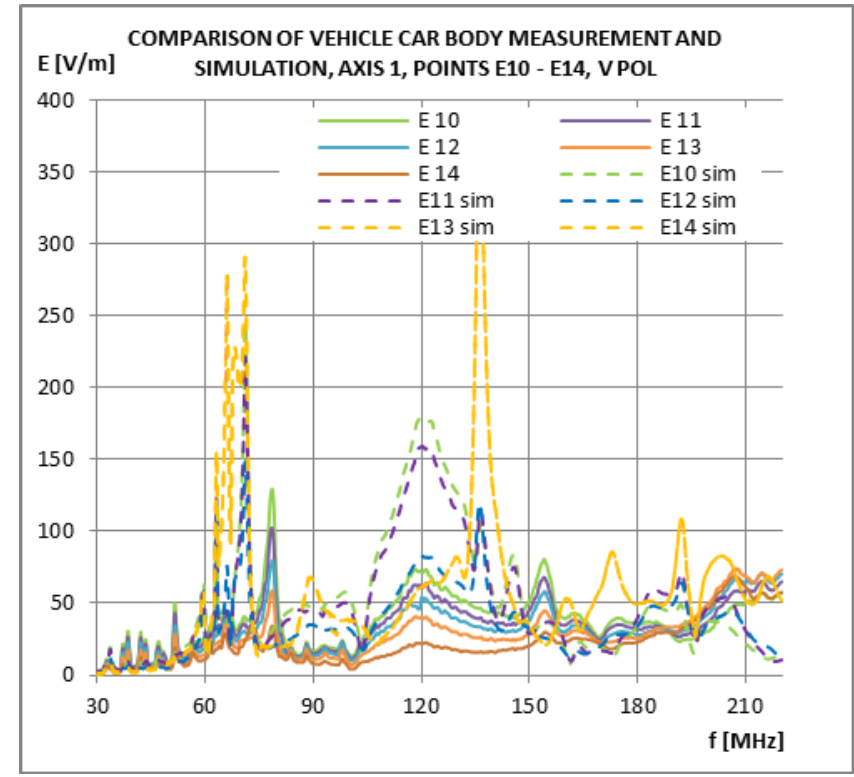

Fig. 14. Comparison of vehicle car body measurement and simulation for points E10 - E14.

sents the value of the electric field in the right-hand interior. Each of these points is additionally located from the electrodynamic point of view in a different part of the dashboard. The point E4 is surrounded by metal reinforcements (dashboard, steering wheel and steering column) on both sides, the point E7 is located near the chrome frame of the infotainment display and the point E12 is located on the plain surface of the dashboard.

\subsection{Comparison of Data Sets}

The average score of all the points compared is shown in Tab. 2. The values below the limit of 0.5 are marked. The course of the individual FSV parameters for point E7 is then shown in Fig. 15 to Fig. 17.

We can notice that a very good degree of compliance of both proposed pre-certification methods has been achieved, especially in the case of vertical polarization of the test field intensity. It is obvious that the proposed numerical simulation achieves good results with pre-certification measurements, especially in the $\mathrm{ADM}_{\mathrm{AV}}$ parameter. The average value across all measured points and polarization $\mathrm{ADM}_{\mathrm{AV}}=0.38$. This result was achieved in particular by setting the parameters of the vehicle simulation model. In the model, the dominant resonance features of the interior trim (dashboard reinforcement and seat structure) are used with the correct choice of sub-mesh locking on the internal stiffeners and body edges.

\begin{tabular}{|c|c|c|c|c|c|c|c|}
\hline $\begin{array}{c}\text { POINT/ } \\
\text { PARAM. }\end{array}$ & $\begin{array}{c}\text { E4 } \\
\text { V }\end{array}$ & $\begin{array}{c}\text { E4 } \\
\text { H }\end{array}$ & $\begin{array}{c}\text { E7 } \\
\text { V }\end{array}$ & $\begin{array}{c}\text { E7 } \\
\text { H }\end{array}$ & $\begin{array}{c}\text { E12 } \\
\text { V }\end{array}$ & $\begin{array}{c}\text { E12 } \\
\text { H }\end{array}$ & TOTAL \\
\hline $\mathrm{ADM}_{\mathrm{AV}}$ & 0.41 & 0.51 & 0.37 & 0.31 & 0.37 & 0.31 & 0.38 \\
\hline $\mathrm{FDM}_{\mathrm{AV}}$ & 0.49 & 0.48 & 0.49 & 0.46 & 0.49 & 0.46 & 0.48 \\
\hline $\mathrm{GDM}_{\mathrm{AV}}$ & 0.70 & 0.74 & 0.67 & 0.58 & 0.68 & 0.58 & 0.66 \\
\hline
\end{tabular}

Tab. 2. Overview of FSV scores at measured points. 
From the ADMi waveforms, it is obvious that the greatest differences between amplitude magnitude obtained by numerical simulation and measurement are in the position of very narrow frequency maxima (absolute difference $|\mathrm{E}|=320 \mathrm{~V} / \mathrm{m}$ at point $\mathrm{E} 4$, vertical polarization). On the contrary, these values are not very high, but we generally see a higher intensity value obtained by numerical simulation. This is due to the modeling of the body as a perfect electric conductor compared to real-body measurements made up of lossy material. It would therefore be possible to consider material differentiation of body parts made up of different types of steels in order to increase the accuracy of the calculation. It is also evident that good results of numerical simulation were given in vertical polarization, when contributions of the frequency maxima with respect to the shape of the cavity are given in particular by their own resonant modes of the body.

Frequency matching $\mathrm{FDM}_{\mathrm{AV}}$ achieves good results with its average value over all measured points and polarization $\mathrm{FDM}_{\mathrm{AV}}=0.48$. Differences between polarizations are not consistent. In the case of vertical polarization, the shapes of the dominant frequency maxima are very high, but their positions are not located identically in both ways.

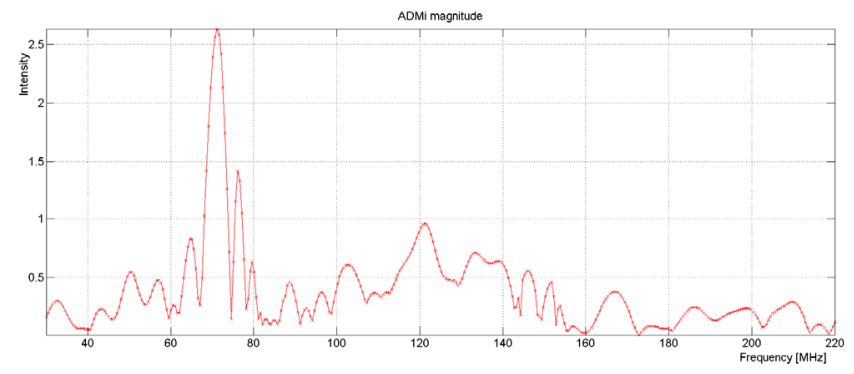

Fig. 15. Frequency dependence of ADMi for point E7 (vertical).

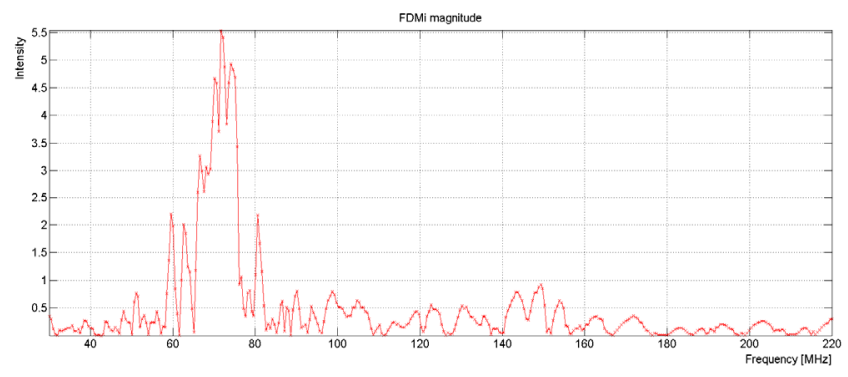

Fig. 16. Frequency dependence of FDMi for point E7 (vertical).

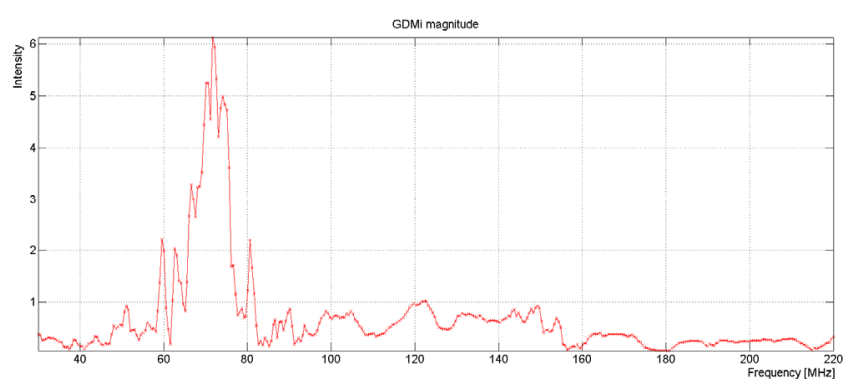

Fig. 17. Frequency dependence of GDMi for point E7 (vertical).
The dominant resonant peak in the $70 \mathrm{MHz}$ band is localized to $72 \mathrm{MHz}$ using numerical methods, while measured at $78 \mathrm{MHz}$. We know that this maximum is caused by the resonance of parts of the steering column and the steering wheel stiffness and is particularly evident in point E4. As a major cause of this frequency difference, we suspect the limited possibility of discretization of the small components constituting this assembly, which in the numerical model according to the rules was modeled by a single piece, which causes the shift of their resonant frequency when exiting different lengths of conductive elements. Conversely, at $120 \mathrm{MHz}$, given in particular by the resonance of the center console reinforcement, both methods are in very good agreement. This part of the car is made from elements whose size can be easily modeled and therefore the resonant lengths for both methods are not different.

The total $\mathrm{GDM}_{\mathrm{AV}}$ compliance level does not reach the required limit at any of the points. The main reason is that the numerical method used and the setting of the simulation model contain conscious shortcomings given by the pre-certification test. It is clear that a perfect model (especially the size of the discretization network elements and a very detailed material description of individual body parts) could be made to increase the level of compliance to a $\mathrm{GDM}_{\mathrm{AV}}$ level of $\leq 0.5$.

\subsection{Proposed Techniques for Improving FSV Compliance}

A key aspect for achieving a high degree of compliance of pre-certification tests and numerical simulations presented with FSV is the correctness of preparing individual datasets for the input method. It is therefore necessary to take the following measures in these areas.

\subsubsection{Numerical Simulation}

1) Obtaining appropriate source data and basic modeling. The most commonly used source data for EMC simulations are crash models in the automotive industry. The basic problems that have to be solved in obtaining a numerical model have been identified.

- Reducing the extreme redundancy of the original model data.

- Removing excess surfaces of the reduced model.

- Appropriate division of the model into functional units.

- Definition of model network size.

- Error correction and control of the resulting model.

2) Preparation of the numerical model. To ensure adequate quality, we set out the basic rules for discretization of the model.

- Respect of phase-dependent distributions of currents and charges along the model structure. The maximum segment size is $1 / 10$ of the shortest wavelength. 
- Using a locally higher density of discretization close to the model and cavity edges.

- Respecting the 1:5 boundary ratio of each triangular segment.

- Increasing local mesh density (sub-mesh) in the vicinity of simulated conductors due to high current and charge gradients.

- Determination of the maximum ratio between the length and diameter of the wire segment (including the thickness of the sheathing) to 3 .

- Ensuring correct connection of triangular segments: segments must not overlap, intersect, and must be connected through common endpoints.

3) Selection of the test field source including its position. We recommend using a vertically and horizontally polarized plane wave in a position from the front of the model. We also recommend simulation from the side of the model (with the same parameters) by horizontal polarization to excite other custom resonant modes of the body cavity.

4) Methods of monitoring and measuring simulation results. It is recommended to monitor the model using electric field intensity distributions, virtual field electric field matrices, current map layouts on the bodywork and virtual voltage and current probe on the harness.

\subsubsection{Simplified Measurement Method}

1) Vehicle selection. To verify numerical simulations, we ideally chose the bare body of a car equipped with parts of the car that have a major influence on the distribution of the electric field strength in the bodywork. Typically, it is the dashboard reinforcement, including the steering wheel and seats (to solve the problem inside the vehicle).

2) Dismantling the car for the test (relevant only when verifying the numerical simulation). A key aspect is the maintenance of a high-quality conductive connection of all used parts of the car.

3) Realization of the pre-certification workplace. Due to the way we perform the radiated electromagnetic field test, we need a shielded workplace. Regarding the high financial demands for the purchase or rental of shielded chambers, we recommend the use of shielded tents.

4) Choice of test method. We recommend using a substitution method to provide the most appropriate access to the test and calibration of the necessary performance.

5) Choice of pre-certification test equipment. We need to carry out the pre-certification test with devices operating on the same principle as the certification exam. However, the choice of cheap and affordable equipment with much lower power and frequency ranges is non-standard. We recommend the use of USB low-cost RF generators and BiLog antennas.

6) Analysis of electric field strength in different models. Apparent differences were observed when performing the test on the bodywork and in an ordinary vehicle. Observing the peak frequencies already found on the bodywork, it has been shown that the bandwidth of these frequency peaks is substantially greater for a production car.

This behavior results from the distinct quality of the resonant cavity, which is significantly higher in the case of the bodywork, as only the conductive parts of the vehicle are located inside the vehicle. On the contrary, the car's space in the production is equipped with a number of plastic, fabric and glass components that significantly reduce the quality of the cavity.

\section{Conclusion}

The article provides a look at the available pre-certification methods of electromagnetic field tests in the automotive industry and compares their information capability with the FSV method.

From the presented findings, the FSV method can be applied without difficulty to the automotive environment, however under the conditions defined in Sec. 4.2.1 and 4.2.2, only relevant input data settings (or the results of the pre-certification procedures) can yield relevant results. The FSV method provided an objective picture of the consistency of both proposed methods.

It follows that both proposed methods capture the frequency position of the maximum in the body quite well. In the case of comparing the amplitude match, worse results have been achieved due to the necessary simplification of the description of body parts and the neglect of elements that cause the local maxima to be attenuated in the real body.

It turns out that the key element in numerical modeling is a thorough model preparation and a good network quality that uniquely determines the accuracy of surface current flow. Without their proper knowledge, it is not possible to correctly identify the distribution of electromagnetic fields in the bodywork using the MoM method to obtain the relevant output of the numerical simulation.

In the case of pre-certification measurements using the modified method, the calibration of the test signal performance plays a key role. The main problem is the frequency gain of the transmitting antenna, which must be covered by a sufficient amplifier power reserve.

The recommended pre-certification methods of measurement are the cheapest implementation of tests not only from the point of view of acquisition and operation of the testing technique, but also of the time used for their preparation and implementation.

\section{Acknowledgments}

Research described in this paper was financed by the Czech Ministry of Education within the framework of the 
National Sustainability Program under grant LO1401. For research, infrastructure of the SIX Center was used.

\section{References}

[1] RUZEK, V., DRINOVSKY, J. Aspects of EMS precompliance testing. In Proceedings of 9th International Conference Vsacký Cáb 2011. Semily, 2011, p. 117-121. ISBN 978-80-214-4319-8

[2] SADIKU, M.N.O. Numerical Techniques in Electromagnetics. 2nd ed. Boca Raton: CRC Press, 2000. ISBN 08-493-1395-3

[3] DRINOVSKY, J., SVACINA, J., RUZEK, V., ZACHAR, J. Electromagnetic compatibility in automotive industry. Elektrorevue - Internet Journal. 2012, vol. 14, no. 3, p.1-8. ISSN: 1213-1539

[4] PIGNARI, S., CANAVERO, F.G. Theoretical assessment of bulk current injection versus radiation. IEEE Transactions on Electromagnetic Compatibility, 1996, vol. 38, no. 3, p. 469-477. DOI: $10.1109 / 15.536077$. ISSN 00189375

[5] ADAMS, J. W., CRUZ, J., MELQUIST, D. Comparison measurements of currents induced by radiation and injection. IEEE Transactions on Electromagnetic Compatibility, 1992, vol. 34, no. 3 , p. $360-362$. DOI: $10.1109 / 15.155856$

[6] ISO 11452-2: Road Vehicles - Component Test Methods for Electrical Disturbances from Narrowband Radiated Electromagnetic Energy - Part 2: Absorber-lined Shielded Enclosure. Ed. 2. Geneva, Switzerland: ISO copyright office, 2004

[7] ISO 11451-4: Road Vehicles - Vehicle Test Methods for Electrical Disturbances from Narrowband Radiated Electromagnetic Energy - Part 4: Bulk Current Injection (BCI). Ed. 3. Geneva, Switzerland: ISO copyright office, 2013.

[8] ISO 11452-4: Road Vehicles - Component Test Methods for Electrical Disturbances from Narrowband Radiated Electromagnetic Energy - Part 4: Harness Excitation Methods. Ed. 4. Geneva, Switzerland: ISO copyright office, 2011.

[9] HILL, D. A. Currents induced on multiconductor transmission lines by radiation and injection. IEEE Transactions on Electromagnetic Compatibility, 1992, vol. 34, no. 4, p. 445-450. DOI: $10.1109 / 15.179277$

[10] COLEBY, D. E., DUFFY, A. P. A visual interpretation rating scale for validation of numerical models. COMPEL: International Journal for Computation and Mathematics in Electrical and Electronic Engineering, 2005, vol. 24, no. 4, p. 1078-1092. DOI: $10.1108 / 03321640510615472$

[11] DUFFY, A. P., MARTIN, A. J. M., ORLANDI, A., et al. Feature Selective Validation (FSV) for validation of computational electromagnetics (CEM). Part I - The FSV method. IEEE Transactions on Electromagnetic Compatibility, 2006, vol. 48, no. 3, p. 449-459. DOI: 10.1109/TEMC.2006.879358

[12] ORLANDI, A., DUFFy, A. P., ARCHAMBEAUlT, B., et al. Feature Selective Validation (FSV) for validation of computational electromagnetics (CEM). Part II - Assessment of FSV performance. IEEE Transactions on Electromagnetic Compatibility, 2006, vol. 48, no. 3, p. 460-467. DOI: 10.1109/TEMC. 2006.879360
[13] JINJUJ, B., ZHANG, G., WANG, L., DUFFY, A. Credibility evaluation of uncertainty analysis results of EMC simulation. In 2014 3rd Asia-Pacific Conference on Antennas and Propagation (APCAP). DOI: 10.1109/APCAP.2014.6992803

[14] JAUREGUI, R., POUS, M., SILVA, F. Use of reference limits in the Feature Selective Validation (FSV) method. In 2014 International Symposium on Electromagnetic Compatibility (EMC Europe). DOI: 10.1109/EMCEurope.2014.6931054

[15] TESAR, J. Gas Permittivity Measurement by Resonator Method. Diploma Thesis. Masaryk University, Brno, 2010. (In Czech)

[16] HARRINGTON, R. F. Field Computation by Moment Methods. Piscataway (USA): IEEE Press, 1993. ISBN 978-0-7803-1014-8

[17] EMCOS, GEORGIA. Harness Studio User's Manual. 162 pages. [Online] Cited 2017-04-25. Available at: www.emcos.com

[18] EMCOS, GEORGIA. EMC Studio: Computer Simulation Software. 130 pages. [Online] Cited 2017-07-28. Available at: https://www.emcos.com/?products=emc-studio

\section{About the Authors ...}

Václav RŮŽEK was born in Tábor, Czech Republic, in 1985. He received his M.Sc. from Electrotechnical Manufacturing and Management in 2009. His research interests include pre-compliance electromagnetic compatibility issues and numerical simulations. He is an EMC specialist in ŠKODA AUTO a.s., responsible for pre-development issues, numerical simulations and he leads region China from the EMC point of view.

Jiří DŘíNOVSKÝ was born in Litomyšl, Czech Republic, in 1979. He received the M.Sc. and Ph.D. degrees in Electronics and Communication from the Brno University of Technology, Brno, Czech Republic, in 2003 and 2007, respectively. His Ph.D. thesis was awarded by Emil Škoda Award in 2007. Since 2006 he has been an assistant professor in Electronics and Communication at the Dept. of Radio Electronics, Brno University of Technology. His research activities include selected topics of EMC, EMI measurements, and EMS testing. He is also interested in specialized problems of radiofrequency and microwave measurements.

Jan CUPÁK was born in Olomouc, in 1986. He received his Master's degree from the Technical University of Brno in 2011. He joined OZM in 2014. He began his career at the company of Chromservis, where he worked in research and development of advanced laboratory equipment designed for CBRN defense. He is experienced in HW design, EMC and the development of new electronic components. Also, he has been responsible for the development of special electronic devices for the measurement of physical properties. 